\title{
Algebras of Local Observables on a Manifold ${ }^{\star}$
}

\author{
J. Dimock ${ }^{\star \star}$ \\ Institute for Advanced Study, Princeton, NJ 08540, USA
}

\begin{abstract}
We propose a generalization of the Haag-Kastler axioms for local observables to Lorentzian manifolds. The framework is intended to resolve ambiguities in the construction of quantum field theories on manifolds. As an example we study linear scalar fields for globally hyperbolic manifolds.
\end{abstract}

\section{Axioms}

Quantum field theories are usually defined on Minkowski space-time, but it seems desirable to generalize to arbitrary Lorentzian manifolds. This is so not only to accommodate physical systems that require a manifold model for spacetime, but also as a means of gaining perspective on the general structure of quantum field theories. General references for quantization on manifolds are De Witt [3] and Isham [12].

The problem can be posed as finding field operators which satisfy given field equations. However, on a general manifold there is no natural choice for the Hilbert space on which the operators act, and, in particular, there is no vacuum state to be used as a reference point. This suggests formulating the problem in terms of the algebraic structure of the field operators, and leaving the specification of states as a secondary step.

One algebraic approach has been developed by Isham [12], Kay [13], and Hajicek [10]. They associate with each Cauchy surface $S$ the $C^{*}$ algebra $\mathscr{A}_{S}$ generated by the canonical commutation relations (CCR) over functions on $S$. The field equations then determine isomorphisms $\mathscr{A}_{S} \rightarrow \mathscr{A}_{\hat{S}}$ which give the dynamics. This type of approach seems quite satisfactory for linear problems, but one can anticipate troubles in extending it to nonlinear problems: things are probably too singular to allow a definition of the algebras $\mathscr{A}_{S}$.

In this paper we propose another algebraic approach which generalizes the Haag-Kastler algebras of local observables on Minkowski space [8]. There is a single $C^{*}$ algebra $\mathscr{A}$ together with distinguished subalgebras $\mathscr{A}(\mathcal{O})$ corresponding to local regions of space-time. All reference to fields to supressed.

* Supported by National Science Foundation PHY 77-21740.

$\star \star$ On leave from Department of Mathematics, SUNY at Buffalo, Buffalo, NY 14214, USA 
We now explain the ideas in detail. We consider a Lorentzian manifold $(\mathscr{M}, g)$ consisting of a four-dimensional manifold $\mathscr{M}$, and a pseudo-Riemannian metric $g$ of signature $(+---)$. We assume that $(\mathscr{M}, g)$ is time-orientable. Then an algebra of local observables on $(\mathscr{M}, g)$ is defined to be a structure satisfying the following five axioms. The first three are essentially the same as those of HaagKastler.

Axiom 1 (Nets of local observables). For each bounded open set $\mathcal{O} \subset \mathscr{M}$ there is a $C^{*}$ algebra $\mathscr{A}(\mathcal{O})$. If $\mathcal{O} \subset \mathcal{O}^{\prime}$ then $\mathscr{A}(\mathcal{O}) \subset \mathscr{A}\left(\mathcal{O}^{\prime}\right)$. The $C^{*}$ algebra $\mathscr{A}=\bigcup \mathscr{A}(\mathcal{O})$ is called the algebra of observables.

Two nets of local observables $\mathscr{A}(\mathcal{O})$ and $\mathscr{A}^{\prime}(\mathcal{O})$ are said to be isomorphic if there is an isomorphism $i: \mathscr{A} \rightarrow \mathscr{A}^{\prime}$ such that $i[\mathscr{A}(\mathcal{O})]=\mathscr{A}^{\prime}(\mathcal{O})$. In accordance with our algebraic point of view we do not distinguish isomorphic nets. If $\mathscr{A}$ is realized as an algebra of operators on a Hilbert space one speaks of a representation of the algebra or the net.

Axiom 2 (Primitivity). $\mathscr{A}$ is primitive.

Primitive means there is a faithful irreducible representation.

Axiom 3 (First causality).

If $\mathcal{O}$ is spacelike separated from $\mathcal{O}^{\prime}$ then $\left[\mathscr{A}(\mathcal{O}), \mathscr{A}\left(\mathcal{O}^{\prime}\right)\right]=0$.

Spacelike separated means that there is no causal curve joining a point in $\mathcal{O}$ to a point in $\mathcal{O}^{\prime}$. A causal curve is a curve whose tangent vectors are either timelike or null.

Axiom 4 (Second causality). If $\mathcal{O}$ is causally dependent on $\mathcal{O}^{\prime}$, then $\mathscr{A}(\mathcal{O}) \subset \mathscr{A}\left(\mathcal{O}^{\prime}\right)$.

The exact meaning of the axiom depends on the definition of causally dependent. A strong form would be to say that $\mathcal{O}$ is causally dependent on $\mathcal{O}^{\prime}$ if every endless causal curve through $\mathcal{O}$ intersects $\mathcal{O}^{\prime}$. Weaker forms may also be adequate.

This axiom embodies the basic dynamical principle that the past determines the future (in a certain local sense). If we know a state on $\mathscr{A}\left(\mathcal{O}^{\prime}\right)$ then we know it on $\mathscr{A}(\mathcal{O})$.

This axiom is not usually included in the Haag-Kastler axioms, although it was proposed by Haag and Schroer [9] at an early date. On Minkowski space it is perhaps not so important because there is another axiom which says that the Poincare transformations define automorphisms of the algebra, and this carries dynamical information. In general we do not have Poincare transformations. There is however a certain covariance which we now explain.

Let us shift our point of view slightly. Instead of requiring a net of local observables $\mathscr{A}(\mathcal{O})$ on a particular $(\mathscr{M}, g)$, we require that there be an $\mathscr{A}(\mathcal{O})$ for each $(\mathscr{M}, g)$ in an isometry class. (Recall that an isometry between $(\mathscr{M}, g)$ and $(\hat{\mathscr{M}}, \hat{g})$ is a diffeomorphism $\kappa: \mathscr{M} \rightarrow \hat{\mathscr{M}}$ such that $\kappa^{*}(\hat{g})=g$.) Isometric space-times are physically equivalent and so we want to impose an equivalence on the associated algebras. Specifically we want the following functorial property:

Axiom 5 (Covariance). For any isometry $\kappa$ from $(\mathscr{M}, g)$ to $(\hat{\mathscr{M}}, \hat{g})$ there is an isomorphism $\alpha_{\kappa}: \mathscr{A} \rightarrow \hat{\mathscr{A}}$ such that $\alpha_{\kappa}[\mathscr{A}(\mathcal{O})]=\hat{\mathscr{A}}(\kappa(\mathcal{O}))$. Furthermore $\alpha_{\text {id }}=$ id and $\alpha_{\kappa_{1}} \circ \alpha_{\kappa_{2}}=\alpha_{\kappa_{10} \kappa_{2}}$. 
If we specialize to the case $(\mathscr{M}, g)=(\hat{\mathscr{M}}, \hat{g})$ the axiom says that for any isometry $\kappa$ on $(\mathscr{M}, g)$ there is an automorphism $\alpha_{\kappa}$ on $\mathscr{A}$ such that $\alpha_{\kappa}[\mathscr{A}(\mathcal{O})]=\mathscr{A}(\kappa(\mathcal{O}))$. In particular if $(\mathscr{M}, g)=$ Minkowski space, the isometries are the Poincare transformations and we recover the usual axiom.

Having explained the axioms let us turn to a discussion of their status. We would like to claim that any quantum system for which a Lorentz manifold model of space-time is appropriate can be described by an algebra of observables in the above sense. Thus we include linear and nonlinear field theories, massive and massless particles, fermions and bosons, etc., etc. This seems to be a reasonable speculation. A deeper question is whether such a structure can provide the basis for a complete description of any system. This does seem to be true on Minkowski space. By extrapolation we might expect that it is true for space-time with special structure, e.g. asymptotically flat or stationary. What is missing in the general case is some idea of how to associate elements of the algebra with specific operations or measurements on the system. Similar difficulties crop up in other quantization schemes.

It would be interesting to further generalize the axioms to a scheme in which the metric is not given, but is somehow a dynamical variable incorporated into the algebra. This problem is discussed by Dyson [5].

\section{Linear Scalar Field}

A. The remainder of the paper is devoted to showing how an algebra of local observables arises naturally for a particular example - the linear scalar field on a globally hyperbolic manifold $(\mathscr{M}, g)$. The program is as follows. We construct a field operator $\phi$ as a solution of the Klein-Gordon equation taking as data a representation $(\theta, \pi)$ of the canonical commutation relations (CCR) over some Cauchy surface $S$. The field operator $\phi$ defines a net of local observables $\mathscr{A}(\mathcal{O})$. Although the field depends on the choice of $S$ and $(\theta, \pi)$, we establish the key result that $\mathscr{A}(\mathcal{O})$ does not. Finally, we show that $\mathscr{A}(\mathcal{O})$ satisfies the axioms.

Our treatment of the linear scalar field follows that of Isham [12]. The main difference (aside from a few technical improvements) lies in our interest in the local algebras. For further results (and earlier references) on the scalar field see Kay [13], Hajicek [10], Wald [18], and Dimock [4]. In another paper we study Dirac fields by similar methods.

B. We begin by defining some function spaces. Let $C^{\infty}(\mathscr{M})$ be the (real-valued) infinitely differentiable functions on $\mathscr{M}$ and $C_{0}^{\infty}(\mathscr{M})$ those with compact support. These are given topologies analogous to the usual topologies for $C^{\infty}\left(\mathbb{R}^{n}\right), \mathbb{C}_{0}^{\infty}\left(\mathbb{R}^{n}\right)$. Every $u \in C^{\infty}(\mathscr{M})$ defines a continuous linear functional on $f \in C_{0}^{\infty}(\mathscr{M})$ by $\langle u, f\rangle=$ $\int u f d V$ where $d V$ is the volume element defined by the metric $g$. Correspondingly we denote the dual space, the distributions, by $\left[C_{0}^{\infty}(\mathscr{M})\right]^{\prime}=C^{-\infty}(\mathscr{M})$. The distributions with compact support are denoted $C_{0}^{-\infty}(\mathscr{M})$ and one can make the usual identification $\left[C^{\infty}(\mathscr{M})\right]^{\prime}=C_{0}^{-\infty}(\mathscr{M})$. (We use the notation of Guillemin and Sternberg [7]; one also writes $C_{0}^{\infty}=\mathscr{D}, C^{\infty}=\mathscr{E}, C^{-\infty}=\mathscr{D}^{\prime}, C_{0}^{-\infty}=\mathscr{E}^{\prime}$.)

We are interested in the Klein-Gordon equation which has the form

$\left(\square+m^{2}\right) u=0$. 
Here the d'Alembertian $\square$ is given in local coordinates by

$$
\square=\nabla^{v} \nabla_{v}=|g|^{-1 / 2} \partial_{\mu} g^{\mu v}|g|^{1 / 2} \partial_{v}
$$

where $|g|=\left|\operatorname{det}\left\{g_{\mu \nu}\right\}\right|$, and $m^{2} \geqq 0$ is a mass parameter. (Our methods also work for any equation of the form $\square u+\langle a, \nabla u\rangle+b u=0$ where $a$ is any smooth vector field on $\mathscr{M}$ and $b$ is any smooth function.)

As a consequence of the global hyperbolicity there are global fundamental solution for $\square+m^{2}$; see Leray [14], Lichnerowicz [15], Choquet-Bruhat [2]. That is there are unique operators $E^{ \pm}: C_{0}^{\infty}(\mathscr{M}) \rightarrow C^{\infty}(\mathscr{M})$ such that

$$
\begin{gathered}
\left(\square+m^{2}\right) E^{ \pm}=E^{ \pm}\left(\square+m^{2}\right)=I \\
\operatorname{supp}\left(E^{ \pm} f\right) \subset J^{ \pm}(\operatorname{supp} f)
\end{gathered}
$$

where for $K \subset \mathscr{M}, J^{ \pm}(K)$ is the set of all points in $\mathscr{M}$ which can be reached from $K$ by a future/past directed causal curve. The d'Alembertian is self-adjoint and correspondingly we may continuously extend $E^{ \pm}$to operators from $C_{0}^{-\infty}(\mathscr{M})$ to $C^{-\infty}(\mathscr{M})$ by $E^{ \pm}=\left(E^{\mp}\right)^{\prime}$. We also define $E=E^{+}-E^{-}$and have $E^{\prime}=-E$.

A Cauchy surface for $\mathscr{M}$ is a spacelike hypersurface $S$ such that any endless causal curve intersects $S$ exactly once. We assume that $\mathscr{M}$ has smooth Cauchy surfaces. (Global hyperbolicity implies the existence of $C^{0}$ Cauchy surfaces [6], [11], but there is no published proof of the existence of $C^{\infty}$ Cauchy surfaces so strictly speaking this is an extra assumption.) A Cauchy surface has the property that $J^{ \pm}(K) \cap J^{\mp}(S)$ is compact for any compact set $K[11]$, [16].

For given $S$ let $\rho_{0}: C^{\infty}(\mathscr{M}) \rightarrow C^{\infty}(S)$ be the restriction operator and let $\rho_{1}: C^{\infty}(\mathscr{M}) \rightarrow C^{\infty}(S)$ be the forward normal derivative.

Theorem 1. (Cauchy problem). Let $S$ be any Cauchy surface and let $u_{0}, u_{1} \in C_{0}^{\infty}(S)$ then there exists a unique $u \in C^{\infty}(\mathscr{M})$ such that $\left(\square+m^{2}\right) u=0, \rho_{0}(u)=u_{0}, \rho_{1}(u)=u_{1}$. Furthermore, supp $u \subset\left(\bigcup_{i} \bigcup_{ \pm} J^{ \pm}\left(\operatorname{supp} u_{i}\right)\right)$.

This is the classical existence and uniqueness thorem. Apparently the existence has never been proved in this form (i.e., arbitrary globally hyperbolic manifold, arbitrary Cauchy surface), although Leray [14] constructs solutions $u \in H_{\text {loc }}^{2}(\mathscr{M})$. We include a proof of the theorem as stated in the Appendix (Lemmas A.2, A.4).

The operators $\rho_{0}, \rho_{1}$ have adjoints $\rho_{0}^{\prime}, \rho_{1}^{\prime}$ which map $C_{0}^{-\infty}(S)$ to $C_{0}^{-\infty}(\mathscr{M})$. Thus $E \rho_{0}^{\prime}$ and $E \rho_{1}^{\prime}$ map $C_{0}^{-\infty}(S)$ to $C^{-\infty}(\mathscr{M})$. As before we have identifications $C^{\infty}(S) \subset C^{-\infty}(S), C_{0}^{\infty}(S) \subset C_{0}^{-\infty}(S)$ defined by $\langle u, f\rangle=\int u f d S$ where $d S$ is the induced Riemannian volume element on $S$. Thus it makes sense to talk of the restriction of these operators to $C_{0}^{\infty}(S)$.

Corollary 1.1 $E \rho_{0}^{\prime}, E \rho_{1}^{\prime}$ restrict to continuous operators from $C_{0}^{\infty}(S)$ to $C^{\infty}(\mathscr{M})$ and

$$
u=E \rho_{0}^{\prime} u_{1}-E \rho_{1}^{\prime} u_{0}
$$

is the solution of the Cauchy problem with data $u_{0}, u_{1}$.

Proof. Let $u$ be the solution with data $u_{0}, u_{1} \in C_{0}^{\infty}(S)$. Then we have the identity 
for $f \in C_{0}^{\infty}(\mathscr{M})$

$$
\langle u, f\rangle=-\left\langle u_{1}, \rho_{0} E f\right\rangle+\left\langle u_{0}, \rho_{1} E f\right\rangle
$$

(see Lemma A.1 in the Appendix). This says that the desired identity $u=E \rho_{0}^{\prime} u_{1}-$ $E \rho_{1}^{\prime} u_{0}$ holds in the sense of distributions. However, specializing to $u_{0}=0$ we see that $E \rho_{0}^{\prime}: C_{0}^{\infty}(S) \rightarrow C^{\infty}(\mathscr{M})$ and specializing to $u_{1}=0$ gives $E \rho_{1}^{\prime}: C_{0}^{\infty}(S) \rightarrow C^{\infty}(\mathscr{M})$. Thus all terms in the identity are $C^{\infty}$ functions and so the identity holds in this sense. The continuity follows by using the closed graph theorem.

Corollary 1.2. $O n C_{0}^{\infty}(S)$ :

$$
\begin{array}{ll}
\rho_{0} E \rho_{0}^{\prime}=0 & \rho_{0} E \rho_{1}^{\prime}=-I \\
\rho_{1} E \rho_{0}^{\prime}=I & \rho_{1} E \rho_{1}^{\prime}=0 .
\end{array}
$$

Proof. Apply $\rho_{0}, \rho_{1}$ to the identity in Corollary 1.1.

Corollary 1.3. On $C_{0}^{\infty}(\mathscr{M})$ :

$$
E=E \rho_{0}^{\prime} \rho_{1} E-E \rho_{1}^{\prime} \rho_{0} E .
$$

Proof. Insert $u_{1}=\rho_{1} u, u_{0}=\rho_{0} u$ in the identity in Corollary 1.1 to get $u=E \rho_{0}^{\prime} \rho_{1} u-$ $E \rho_{1}^{\prime} \rho_{0} u$ for any solution $u$ such that $\rho_{0} u, \rho_{1} u$ have compact support. Now take $u=E f .\left(\rho_{0} E f, \rho_{1} E f\right.$ have compact support since $S \cap J^{ \pm}$(supp $\left.f\right)$ is compact.)

C. Now we turn to the quantum problem. For any Cauchy surface $S$ we consider representations of the CCR over $S$. These will consist of a complex Hilbert space $\mathscr{H}$ and symmetric operators $\theta(h), \pi(h)$, linear in $h \in C_{0}^{\infty}(S)$, defined on a dense domain in $\mathscr{H}$, and satisfying

$\left[\theta(h), \pi\left(h^{\prime}\right)\right]=i\left\langle h, h^{\prime}\right\rangle=i \int h h^{\prime} d S$.

The existence of representations is easily established. Let $\mathscr{H}_{1}$ be the completion of complex valued $C_{0}^{\infty}(S)$ with inner product $\left(h, h^{\prime}\right)=\left\langle\bar{h}, h^{\prime}\right\rangle$, let $\mathscr{H}_{0}=\mathbb{C}, \mathscr{H}_{n}=$ $\underset{\text { sym }}{\otimes} \mathscr{H}_{1}$, and let $\mathscr{H}=\bigoplus_{n=1}^{\infty} \mathscr{H}_{n}$ be the Fock space. If $a(h), a^{*}(h)$ are the usual creation and annihilation operators defined on finite particle vectors in $\mathscr{H}$ and satisfying $\left[a(h), a^{*}\left(h^{\prime}\right)\right]=\left\langle h, h^{\prime}\right\rangle$, then

$$
\begin{aligned}
& \theta(h)=\left(a^{*}(h)+a(h)\right) / \sqrt{2} \\
& \pi(h)=i\left(a^{*}(h)-a(h)\right) / \sqrt{2}
\end{aligned}
$$

provide a representation of the $C C R$ over $S$. We do not attach any special significance to this representation.

We now mimic the classical Cauchy problem.

Theorem 2. Let $(\theta, \pi)$ be a representation of the CCR over a Cauchy surface $S \subset \mathscr{M}$. Define a field operator in the sense of distribution by

$$
\phi=E \rho_{0}^{\prime} \pi-E \rho_{1}^{\prime} \theta \text {. }
$$

Then $\phi$ solves the Klein-Gordon equation

$$
\left(\square+m^{2}\right) \phi=0
$$


and

$\left[\phi(f), \phi\left(f^{\prime}\right)\right]=1 / i\left\langle f, E f^{\prime}\right\rangle$.

Proof. The operator $\phi(f)$ is defined for $f \in C_{0}^{\infty}(\mathscr{M})$ by

$$
\phi(f)=\theta\left(\rho_{1} E f\right)-\pi\left(\rho_{0} E f\right) .
$$

This makes sense since $\rho_{0} E f, \rho_{1} E f \in C_{0}^{\infty}(S)$. The field equation $\phi\left(\left(\square+m^{2}\right) f\right)=0$ follows from $E\left(\square+m^{2}\right)=0$. Finally

$$
\left[\phi(f), \phi\left(f^{\prime}\right)\right]=-i\left\langle\rho_{1} E f, \rho_{0} E f^{\prime}\right\rangle+i\left\langle\rho_{0} E f, \rho_{1} E f^{\prime}\right\rangle
$$

which is $1 / i\left\langle f, E f^{\prime}\right\rangle$ by Corollary 1.3 .

Corollary 2.1. Let $\hat{S}$ be another Cauchy surface in $\mathscr{M}$ and let $\hat{\theta}, \hat{\pi}$ be the restrictions of $\phi$ to $\hat{S}$ defined as distributions by

$$
\begin{aligned}
& \hat{\theta}=\hat{\rho}_{0} \phi=\hat{\rho}_{0} E \rho_{0}^{\prime} \pi-\hat{\rho}_{0} E \rho_{1}^{\prime} \theta \\
& \hat{\pi}=\hat{\rho}_{1} \phi=\hat{\rho}_{1} E \rho_{0}^{\prime} \pi-\hat{\rho}_{1} E \rho_{1}^{\prime} \theta .
\end{aligned}
$$

Then $(\hat{\theta}, \hat{\pi})$ provide a representation of the CCR over $\hat{S}$. If $\hat{S}=S$ then $\hat{\theta}=\theta, \hat{\pi}=\pi$. Proof. The definition says $\hat{\theta}(h)=\theta\left(\rho_{1} E \rho_{0}^{\prime} h\right)+\ldots$ and makes sense since $\rho_{1} E \hat{\rho}_{0}^{\prime}: C_{0}^{\infty}(\hat{S}) \rightarrow C_{0}^{\infty}(S)$, etc. For the commutator we have:

$$
\begin{aligned}
{\left[\hat{\theta}(h), \hat{\pi}\left(h^{\prime}\right)\right]=} & -i\left\langle\rho_{1} E \hat{\rho}_{0}^{\prime} h, \rho_{0} E \hat{\rho}_{1}^{\prime} h^{\prime}\right\rangle \\
& +i\left\langle\rho_{0} E \hat{\rho}_{0}^{\prime} h, \rho_{1} E \hat{\rho}_{1}^{\prime} h^{\prime}\right\rangle
\end{aligned}
$$

But Green's identity gives that for solutions $u, u^{\prime}$, the quantity $\left\langle\rho_{1} u, \rho_{0} u^{\prime}\right\rangle-$ $\left\langle\rho_{0} u, \rho_{1} u^{\prime}\right\rangle$ is independent of $S$. Thus we may change $\rho_{0}, \rho_{1} \rightarrow \hat{\rho}_{0}, \hat{\rho}_{1}$ above. Then by Corollary 1.2 we get $i\left\langle h, h^{\prime}\right\rangle$ as required. The result for $\hat{S}=S$ also follows by Corollary 1.2.

D. Next we introduce the algebras. First we pass to the Weyl form of the $C C R$. Formally if

$$
W\left(h, h^{\prime}\right)=\exp \left(i\left(\theta(h)-\pi\left(h^{\prime}\right)\right)\right)
$$

we have

$$
W\left(h_{1}, h_{1}^{\prime}\right) W\left(h_{2}, h_{2}^{\prime}\right)=W\left(h_{1}+h_{2}, h_{1}^{\prime}+h_{2}^{\prime}\right) \exp \left(-\frac{i}{2}\left(\left\langle h_{1}, h_{2}^{\prime}\right\rangle-\left\langle h_{2}, h_{1}^{\prime}\right\rangle\right)\right) .
$$

This can be made rigorous for the example we gave. In any case, we now define a representation of the $C C R$ over $S$ to be a function $W$ from $C_{0}^{\infty}(S) \times C_{0}^{\infty}(S)$ to unitary operators on a Hilbert space $\mathscr{H}$ such that the above identity is satisfied. We also require $t \rightarrow W\left(t h, t h^{\prime}\right)$ is strongly continuous. Then by Stone's theorem one can define self-adjoint $\theta(h), \pi\left(h^{\prime}\right)$ so $e^{i \theta(h) t}=W(t h, 0)$ and $e^{-i \pi\left(h^{\prime}\right) t}=W\left(0, t h^{\prime}\right)$ and one can show that these form a representation of the $C C R$ in the previous sense. Thus the new notion generalizes the old.

Given a representation in the above sense we now define a self-adjoint field operator $\phi(f), f \in C_{0}^{\infty}(\mathscr{M})$, via Stone's theorem by

$$
e^{i \phi(f) t}=W\left(t \rho_{1} E f, t \rho_{0} E f\right) .
$$


We define a net of local observables by

$$
\mathscr{A}(\mathcal{O})=C^{*} \text { algebra generated by } e^{i \phi(f)}=W\left(\rho_{1} E f, \rho_{0} E f\right), \operatorname{supp} f \subset \mathcal{O}
$$

and define

$$
\mathscr{A}=\overline{\bigcup_{\mathcal{O}} \mathscr{A}(\mathcal{O})}=\mathscr{A}(\mathscr{M})
$$

The algebra $\mathscr{A}$ can also be characterized as the $C^{*}$ algebra generated by $W\left(h, h^{\prime}\right)$, $h, h^{\prime} \in C_{0}^{\infty}(S)$. Clearly the latter contains the former. For the inclusion the other way suppose we are given $h, h^{\prime}$, let $u$ be the solution with data $h, h^{\prime}$, and choose $f$ so $u=E f$ (see Lemma A.3). Then $W\left(h, h^{\prime}\right)=W\left(\rho_{1} E f, \rho_{0} E f\right)$ which gives the inclusion.

Theorem 3. The net $\mathscr{A}(\mathcal{O})$ is independent of the representation $(\mathscr{H}, W)$ of the $C C R$ over $C_{0}^{\infty}(S)$, and is independent of the Cauchy surface $S$.

Proof. For given $S$ we consider two representations $(\mathscr{H}, W)$ and $(\tilde{\mathscr{H}}, \tilde{W})$ over $C_{0}^{\infty}(S)$ and the associated nets $\mathscr{A}(\mathcal{O}), \tilde{A}(\mathcal{O})$. We must show there is an isomorphism $i: \mathscr{A} \rightarrow \tilde{\mathscr{A}}$ such that $i[\mathscr{A}(\mathcal{O})]=\mathscr{A}(\mathcal{O})$. Different representations of the $C C R$ give rise to isomorphic $C^{*}$ algebras, a result due to Manuceau and Slawny (see references in [10], [12] or Simon [17]). Since $\mathscr{A}, \tilde{\mathscr{A}}$ are generated by $W, \tilde{W}$ this says that there is an $i: \mathscr{A} \rightarrow \mathscr{\mathscr { A }}$ such that $i\left[W\left(h, h^{\prime}\right)\right]=\tilde{W}\left(h, h^{\prime}\right)$. But this entails $i\left[e^{i \phi(f)}\right]=$ $e^{i \tilde{\phi}(f)}$ and hence $i[\mathscr{A}(\mathcal{O})]=\tilde{\mathscr{A}}(\mathcal{O})$.

Now consider two Cauchy surfaces $S, \hat{S}$. To show that they give rise to isomorphic nets it suffices to find particular representations $(\mathscr{H}, W)$ over $C_{0}^{\infty}(S)$ and $(\hat{\mathscr{H}}, \hat{W})$ over $C_{0}^{\infty}(\hat{S})$ such that the nets are identical. Take any $(\mathscr{H}, W)$ we define $\hat{\mathscr{H}}=\mathscr{H}$ and $\hat{W}$ as in Corollary 2.1 by

$$
\hat{W}\left(h, h^{\prime}\right)=W\left(\rho_{1} E \hat{\rho}_{0}^{\prime} h-\rho_{1} E \hat{\rho}_{1}^{\prime} h^{\prime} . \rho_{0} E \hat{\rho}_{0}^{\prime} h-\rho_{0} E \hat{\rho}_{1}^{\prime} h^{\prime}\right) \text {. }
$$

That this is a representation follows as in Corollary 2.1. Then by Corollary 1.3:

$$
\begin{aligned}
e^{i \hat{\phi}(f)} & =\hat{W}\left(\hat{\rho}_{1} E f, \hat{\rho}_{0} E f\right) \\
& =W\left(\rho_{1} E f, \rho_{0} E f\right) \\
& =e^{i \phi(f)} .
\end{aligned}
$$

Therefore $\hat{\mathscr{A}}(\mathcal{O})=\mathscr{A}(\mathcal{O})$ and the proof is complete.

Now we come to the main result.

Theorem 4. For any globally hyperbolic space-time $(\mathscr{M}, g)$ let $\mathscr{A}(\mathcal{O})$ be the net of local observables defined above. Then $\mathscr{A}(\mathcal{O})$ satisfies the axioms 1-5.

Proof. 1. is established. 2. follows since $C^{*}$ algebras generated by the $C C R$ are primitive. For 3 note that

$$
e^{i \phi(f)} e^{i \phi\left(f^{\prime}\right)}=e^{i \phi\left(f^{\prime}\right)} e^{i \phi(f)} e^{\left\langle f, E f^{\prime}\right\rangle} .
$$

If $\operatorname{supp} f$ and $\operatorname{supp} f^{\prime}$ cannot be joined by a causal curve then $\left\langle f, E f^{\prime}\right\rangle=0$ and so $\left[e^{i \phi(f)}, e^{i \phi\left(f^{\prime}\right)}\right]=0$ which gives the result. 
For axiom 4 we must say what is meant by " $\mathcal{O}$ is causally dependent on $\mathcal{O}^{\prime}$." By this we will mean that there is a Cauchy surface $S$ such that every endless causal curve through $x \in \mathcal{O}$ intersects $S$ in $\mathcal{O}^{\prime}$. When this is the case we must show $\mathscr{A}(\mathcal{O}) \subset \mathscr{A}\left(\mathcal{O}^{\prime}\right)$. We work in a representation based on $S$ with $e^{i \phi(f)}=W\left(\rho_{1} E f, \rho_{0} E f\right)$. If $\operatorname{supp} f \subset \mathcal{O}$, then

$$
\operatorname{supp}\left(\rho_{i} E f\right) \subset\left(\bigcup^{ \pm}(\operatorname{supp} f) \cap S\right) \subset \mathcal{O}^{\prime}
$$

By Lemma A.3, there exists $f^{\prime} \in C_{0}^{\infty}\left(\mathcal{O}^{\prime}\right)$ such that $E f=E f^{\prime}$. Then $e^{i \phi(f)}=e^{i \phi\left(f^{\prime}\right)}$, hence $e^{i \phi(f)} \in \mathscr{A}\left(\mathcal{O}^{\prime}\right)$, and hence $\mathscr{A}(\mathcal{O}) \subset \mathscr{A}\left(\mathcal{O}^{\prime}\right)$.

For axiom 5 we suppose that $\kappa:(\mathscr{M}, g) \rightarrow(\hat{\mathscr{M}}, \hat{g})$ and show that we can find representations such that $\mathscr{A}=\mathscr{A}, \mathscr{A}(\mathcal{O})=\hat{\mathscr{A}}(\kappa(\mathcal{O}))$. This will determine an isomorphism in any other representation. Let $(\mathscr{H}, W)$ be a representation over $C_{0}^{\infty}(S)$ for some Cauchy surface $S \subset \mathscr{M}$. Define a representation $(\hat{\mathscr{H}}, \hat{W})$ over $C_{0}^{\infty}(\hat{S}), \hat{S} \subset \hat{\mathscr{M}}$ by $\hat{S}=\kappa(S), \hat{\mathscr{H}}=\mathscr{H}$, and

$$
\hat{W}\left(h, h^{\prime}\right)=W\left(\kappa_{0}^{*} h, \kappa_{0}^{*} h^{\prime}\right)
$$

where $\kappa_{0}: S \rightarrow \hat{S}$ is the induced diffeomorphism. This is easily checked to be a representation using $\left\langle\kappa_{0}^{*} h, \kappa_{0}^{*} h^{\prime}\right\rangle=\left\langle h, h^{\prime}\right\rangle$. From $\kappa^{*} \hat{\square}=\square \kappa^{*}$ and the uniqueness of the fundamental solutions we conclude $\kappa^{*} \hat{E}^{ \pm}=E^{ \pm} \kappa^{*}$. Using also $\hat{\kappa}_{0}^{*} \hat{\rho}_{i}=$ $\rho_{i} \kappa^{*}$ we have

$$
\begin{aligned}
e^{i \hat{\phi}(f)} & =\hat{W}\left(\hat{\rho}_{1} \hat{E} f, \hat{\rho}_{0} \hat{E} f\right) \\
& =W\left(\rho_{1} E \kappa^{*} f, \rho_{0} E \kappa^{*} f\right) \\
& =e^{i \phi\left(\kappa^{*} f\right)}
\end{aligned}
$$

Thus $\hat{\mathscr{A}}(\mathcal{O})=\mathscr{A}\left(\kappa^{-1}(\mathcal{O})\right)$ or $\mathscr{A}(\mathcal{O})=\hat{\mathscr{A}}(\kappa(\mathcal{O}))$ as required. The remaining properties are straightforward to check.

\section{Appendix}

In this Appendix we prove the existence and uniqueness theorem for the Cauchy problem as stated in Theorem I, and in the process obtain some results used elsewhere in the paper. As in the text $(\mathscr{M}, g)$ is a globally hyperbolic Lorentzian manifold, $E^{ \pm}$are fundamental solutions for $\left(\square+m^{2}\right), E=E^{+}-E^{-}, S$ is a Cauchy surface in $\mathscr{M}, \rho_{0}$ is restriction to $S$, and $\rho_{1}$ is the forward normal derivative on $S$. The first two Lemmas are standard.

Lemma A.1. Let $u$ be a $C^{\infty}$ solution of $\left(\square+m^{2}\right) u=0$ on $\mathscr{M}$ and set $u_{0}=\rho_{0}(u)$, $u_{1}=\rho_{1}(u)$. Then for any $f \in C_{0}^{\infty}(\mathscr{M})$,

$$
\int_{\mathscr{M}} u f d V=\int_{S}\left(u_{0} \rho_{1}(E f)-u_{1} \rho_{0}(E f)\right) d S .
$$

Proof. Green's identity has the form

$$
\begin{aligned}
& \int_{D}\left(u\left(\square+m^{2}\right) v-v\left(\square+m^{2}\right) u\right) d V \\
& \quad=\int_{\partial D}\left(u \frac{\partial v}{\partial n}-v \frac{\partial u}{\partial n}\right) d S
\end{aligned}
$$


where $\partial / \partial n$ is the outward normal derivative on $\partial D$. We let $u$ be the solution, put $v=E^{+} f$, and take $D=S^{-} \equiv J^{-}(S) \backslash S$ so that $\partial D=S$ and $\rho_{1}=\partial / \partial n$. Since $J^{+}(\operatorname{supp} f) \cap J^{-}(S)$ is compact the integrals are well-defined and the identity holds. Thus we have

$$
\int_{S^{-}} u v d V=\int_{S}\left(u_{0} \rho_{1}\left(E^{+} v\right)-u_{1} \rho_{0}\left(E^{+} v\right)\right) d S
$$

Similarly with $v=E^{-} f, D=S^{+} \equiv J^{+}(S) \backslash S$ and $\rho_{1}=-\partial / \partial n$ :

$$
\int_{S^{+}} u v d V=-\int_{S}\left(u_{0} \rho_{1}\left(E^{-} v\right)-u_{1} \rho_{0}\left(E^{-} v\right)\right) d S
$$

Adding these equations gives the result.

Lemma A.2. (a) (Uniqueness) If $\left(\square+m^{2}\right) u=0, \rho_{0}(u)=0, \rho_{1}(u)=0$, then $u=0$

(b) (Support) If $\left(\square+m^{2}\right) u=0$ and $\rho_{0}(u), \rho_{1}(u)$ have support in $N \subset S$ then

$$
\text { supp } u \subset J^{+}(N) \cup J^{-}(N)
$$

Proof. (a) By Lemma A.1, $\int u f d V=0$ for all $f$ and so $u=0$.

(b) If supp $f \subset \sim\left(J^{+}(N) \cup J^{-}(N)\right)$ then there is no causal curve from supp $f$ to $N$. Therefore $\operatorname{supp} E f \cap N=\varnothing$ and by Lemma A.1 $\int u f d V=0$.

Lemma A.3. Let $u$ be a solution of $\left(\square+m^{2}\right) u=0$ and let $\rho_{0}(u), \rho_{1}(u)$ have support in a compact subset $N$ of $S$. Then for any open neighborhood $\mathcal{O}$ of $N$ there exists $f \in C_{0}^{\infty}(\mathscr{M})$ such that supp $f \subset \mathcal{O}$ and $u=E f$.

Remarks. This is a refined version of a result due to Choquet-Bruhat [1]. For the proof we use the fact that if $\left(\square+m^{2}\right) u=f, f \in C_{0}^{\infty}(\mathscr{M})$, and supp $u$ is compact to the past/future then $u=E^{ \pm} f[15]$. A set $A$ is called compact to the past/future if $J^{\mp}(x) \cap A$ is compact for all $x$. Examples: $S$, compact $K, J^{+}(K)$ are compact to the past.

Proof. We may assume $\mathcal{O}$ has a compact closure. Let $\mathcal{O}^{ \pm}=\mathcal{O} \cup S^{ \pm}$and let $\mathcal{O}^{0}=$ $\sim\left(J^{+}(N) \cup J^{-}(N)\right)$. Then $\mathcal{O}^{ \pm}, \mathcal{O}^{0}$ form an open covering of $\mathscr{M}$, and we let $\phi^{0}, \phi^{ \pm}$ be a partition of unity subordinate to the covering. By Lemma A.2 $\phi^{0} u=0$ and so $u=\phi^{+} u+\phi^{-} u$. We define

$$
f=\left(\square+m^{2}\right) \phi^{+} u=-\left(\square+m^{2}\right) \phi^{-} u
$$

Then $\operatorname{supp} f \subset \mathcal{O}^{+} \cap \mathcal{O}^{-}=\mathcal{O}$. Furthermore since $\operatorname{supp}\left(\phi^{ \pm} u\right)$ is compact to the past/future we have $\phi^{ \pm} u= \pm E^{ \pm} f$ and hence $u=E f$.

Lemma A.4. (Existence) For any $u_{0}, u_{1} \in C_{0}^{\infty}(S)$ there exists $u \in C^{\infty}(\mathscr{M})$ such that $\left(\square+m^{2}\right) u=0, \rho_{0}(u)=u_{0}, \rho_{1}(u)=u_{1}$.

Proof. We begin with a local existence theorem. For any point $p \in S$ choose a neighborhood with coordinates $x^{\mu}$ such that $x^{\mu}(p)=0$ and $S$ corresponds to $x^{0}=0$. Let $\mathcal{N}$ be the open lens-shaped region bounded by the hypersurfaces

$$
\begin{aligned}
& x^{0}= \pm\left(\varepsilon^{2}-\left(x^{1}\right)^{2}-\left(x^{2}\right)^{2}-\left(x^{3}\right)^{2}\right) \\
& \left(x^{1}\right)^{2}+\left(x^{2}\right)^{2}+\left(x^{3}\right)^{2} \leqq \varepsilon^{2}
\end{aligned}
$$

For $\varepsilon$ sufficiently small these boundary surfaces will be space-like, as well as the $x^{0}=$ constant surfaces in $\mathscr{N}$. Then by using the Cauchy-Kowaleski theorem 
and energy estimates (e.g., [11], Sect. 7.2) one can show that for $u_{0}, u_{1} \in C_{0}^{\infty}(\mathcal{N} \cap S)$ there exists $u \in C^{\infty}(\mathcal{N})$ such that $\left(\square+m^{2}\right) u=0, \rho_{0}(u)=u_{0}, \rho_{1}(u)=u_{1}$.

Next we obtain a global solution for local data. That is for any $p \in S$ there is an open neighborhood $N \subset S$ of $p$ such that for $u_{0}, u_{1} \in C_{0}^{\infty}(S), \operatorname{supp} u_{0}, \operatorname{supp} u_{1} \subset N$, there is $u \in C^{\infty}(\mathscr{M})$ so that $\left(\square+m^{2}\right) u=0, \rho_{0}(u)=u_{0}, \rho_{1}(u)=u_{1}$. To see this take a neighborhood $\mathcal{N}$ of $p$ as above let $N=\mathscr{N} \cap S$, and at first let $u$ be the solution in $\mathscr{N}$. Now $\mathscr{N}$ is itself a globally hyperbolic manifold (with Cauchy surface $N$ ) and so has fundamental solutions $E_{\mathscr{N}}^{ \pm}$. Then by Lemma A.3 we may write $u=E_{\mathscr{N}} f$ for some $f \in C_{0}^{\infty}(\mathscr{N})$. Now extend $u$ to $\mathscr{M}$ by regarding $f$ as an element of $C_{0}^{\infty}(\mathscr{M})$ and defining $u=E f$. This is an extension since $E^{ \pm} f$ restricted to $\mathscr{N}$ satisfies $\left(\square+m^{2}\right) u=f$ and is compact to the past/future and hence equals $E_{\mathscr{N}}^{ \pm} f$. The extended $u$ is a solution and has the correct data on $N$. Furthermore $u$ vanishes on $S \backslash N$ since supp $u \subset J^{+}(\mathscr{N}) \cup J^{-}(\mathscr{N})$ which does not intersect $S \backslash N$.

Finally we take $u_{0}, u_{1} \in C_{0}^{\infty}(S)$. Let $\left\{N_{k}\right\}$ be a finite open covering of supp $u_{0} \cup$ $\operatorname{supp} u_{1}$ by sets for which global solutions exist, let $\left\{\phi_{k}\right\}$ be a partition of unity subordinate to the covering, and let $u^{k}$ be the solution with data $\phi_{k} u_{0}, \phi_{k} u_{1}$. Then $u=\sum_{k} u^{k}$ is a global solution with data $u_{0}, u_{1}$.

\section{References}

1. Fourés-Bruhat, Y. : Propagateurs et solutions d'equations homogénes hyperboliques. C. R. Acad. Sci. 251, 29 (1960)

2. Choquet-Bruhat, Y.: Hyperbolic differential equations on a manifold. In: Batelle rencontres (eds. C. De Witt, J. Wheeler). New York : Benjamin 1977

3. De Witt, B. : Quantum field theory in curved space-time. Phys. Rep. 19C 295 (1974)

4. Dimock, J. : Scalar quantum field in an external gravitational field. J. Math. Phys. (N. Y.) 20, 2549 (1979)

5. Dyson, F. : Missed opportunities. Bull. Am. Math. Soc. 78, 635 (1972)

6. Geroch, R. : The domain of dependence. J. Math. Phys. (N. Y.) 11, 437 (1970)

7. Guillemin, V., Sternberg, S. : Geometric asymptotics, Providence, R.I. : Amer. Math. Soc. 1977

8. Haag, R., Kastler, D. : An algebraic approach to quantum field theory. J. Math. Phys. 5, 848 (1964)

9. Haag, R., Schroer, S. : Postulates of quantum field theory, J. Math. Phys. 3, 248 (1962)

10. Hajicek, P. : Observables for quantum fields on curved backgrounds. In: Differential geometrical methods in mathematical physics II (eds. K. Bleuler, H. Petry, A. Reetz), p. 535. Berlin, Heidelberg, New York: Springer 1978

11. Hawking, S., Ellis, G. : The large scale structure of space-time. Cambridge: Cambridge University Press, 1973

12. Isham, C. : Quantum field theory in curved space-time: a general mathematical framework. In: Differential geometrical methods in mathematical physics II (eds. K. Bleuler, H. Petry, A. Reetz), p. 459. Berlin, Heidelberg, New York: Springer 1978

13. Kay, B. : Linear spin-zero quantum fields in external gravitational and scalar fields: I: Commun. Math. Phys. 62, 55 (1978); II : Commun. Math. Phys. 71, 29, (1980)

14. Leray, J. : Hyperbolic differential equations. Lecture notes, Princeton 1953 (unpublished)

15. Lichnerowicz, A. : Propagateurs et commutateurs en relativité général. Publication IHES, no. 10, (1961)

16. Penrose, R. : Techniques of differential topology in relativity. SIAM, Philadelphia (1972)

17. Simon, B.: Topics in functional analysis. In: Mathematics of contemporary physics (ed. R. Streater). New York: Academic Press 1972

18. Wald, R. : Existence of the S-matrix in quantum field theory in curved space-time. Ann. Phys. (N. Y.) 118, 490 (1979)

Communicated by R. Haag

Received January 23, 1980 